\title{
Fatal disseminated fusarium infection in acute lymphoblastic leukaemia in complete remission
}

\author{
B Austen, H McCarthy, B Wilkins, A Smith, A Duncombe
}

\begin{abstract}
Fusarium species are increasingly recognised as serious pathogens in the immunocompromised. The outcome in the context of persistent severe neutropenia has been almost universally fatal. However, there have been several case reports of successful treatment if neutrophil recovery can be achieved. This report presents the case of a fatality that occurred despite neutrophil recovery. A 67 year old man developed disseminated fusariosis during the neutropenic phase of induction chemotherapy for acute lymphoblastic leukaemia. Fusarium dimerum was isolated from blood cultures. This species is highly unusual and very few case reports exist in the literature. An initial response to amphotericin treatment coincided with neutrophil recovery but a subsequent relapse occurred, despite adequate neutrophil counts, which proved fatal. It is postulated that reseeding of the blood from an occult site, namely the right vitreum in this case, led to this secondary relapse despite achieving complete leukaemic remission. (f Clin Pathol 2001;54:488-490)
\end{abstract}

Keywords: fusarium; disseminated; neutropenia; remission

Department of

Haematology,

Southampton General

Hospital, Tremona

Road, Southampton

S016 6YD, UK

B Austen

H McCarthy

A Smith

A Duncombe

Department of

Histopathology,

Southampton General

Hospital

B Wilkins

Correspondence to: Dr Austen, 56 Lyric Way, Thornhill, Cardiff CF14 9BP, UK

Belindadoc@aol.com

Accepted for publication 15 March 2001

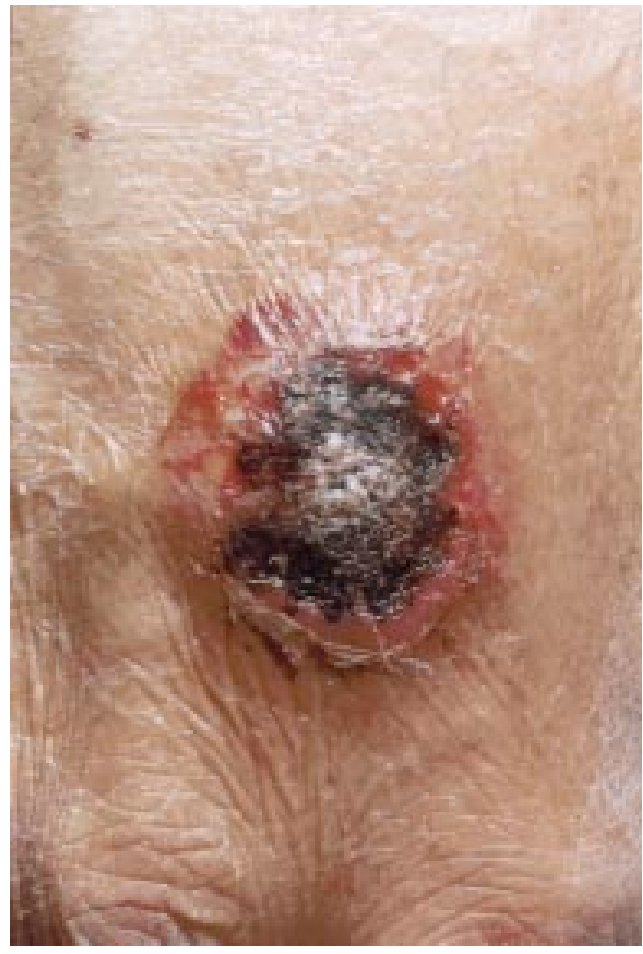

Figure 1 Initial lesion on dorsum of right hand, which developed an ecthyema gangrenosum appearance.
A 67 year old man presented with a three to four week history of general malaise, weight loss, intermittent high fevers, and spontaneous bruising. An initial full blood count demonstrated neutropenia and thrombocytopenia, and subsequent investigations confirmed a diagnosis of acute lymphoblastic leukaemia (ALL). He was treated on the MRC UK ALL 12 induction protocol, which includes high dose steroids. This was given via a tunnelled intravenous catheter. Fluconazole was given from day 1 and the patient was nursed on an open ward without HEPA (high efficiency particulate air) filtration. His absolute neutrophil count fell to zero by day 5 of treatment.

On day 22 during absolute neutropenia, the patient developed a painful macule on the dorsum of his right hand. Over the next few days this evolved, developing a necrotic centre and an ecthyema gangrenosum-like appearance (fig 1). He remained afebrile but was treated with broad spectrum antibiotics. New lesions subsequently appeared on his face, trunk, and limbs, initially appearing as erythematous macules and subsequently developing into flaccid vesicles and then into necrotic lesions (fig 2). Empirical intravenous conventional amphotericin was commenced. Blood cultures subsequently grew Fusarium dimerum and so the

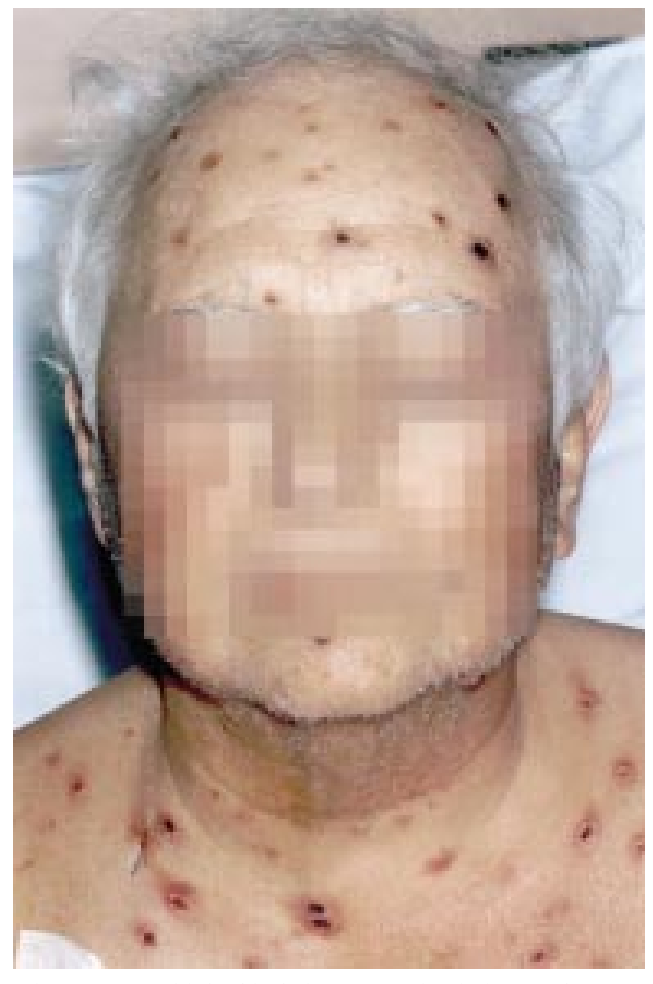

Figure 2 Multiple skin lesions on patient's face and thorax. Initially, many had a vesicular appearance and later developed central necrosis. 


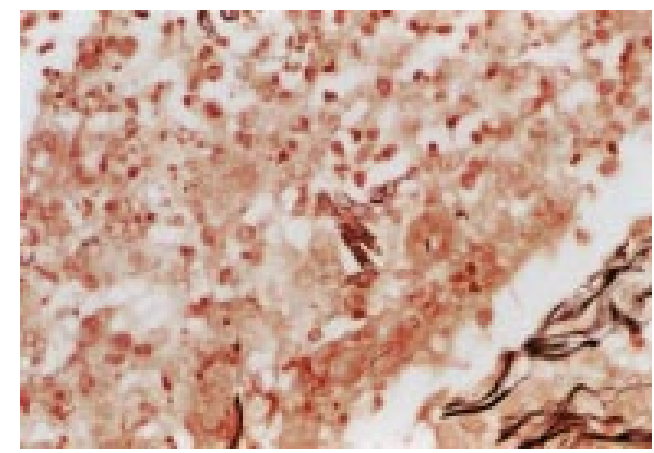

Figure 3 Reticulin stain of skin biopsy showing fungal hyphae.

antifungal treatment was changed to high dose liposomal amphotericin (Ambisome) at a dose of $3 \mathrm{mg} / \mathrm{kg} /$ day. Susceptibility testing later confirmed sensitivity to amphotericin with resistance to itraconazole. In addition, treatment with granulocyte colony stimulating factor ( $\mathrm{G}-\mathrm{CSF}$ ) was started. A bone marrow biopsy was performed, which confirmed the clearance of leukaemic blasts, but with minimal haematopoiesis. The patient remained unwell until day 31 when neutrophil recovery began to occur. At this stage, his skin lesions started to heal and his general condition improved. Repeat sets of blood cultures were negative. Three days later the patient reported a loss of vision in his right eye. Opthalmological assessment demonstrated a superonasal white mass in the right vitreum presumed to be fungus, with an associated chorioretinitis. A right vitrectomy was performed and local intravitreal amphotericin commenced. An improvement in visual acuity was seen. However, a few days later the patient's overall condition began to deteriorate despite having an adequate neutrophil count, continuing with systemic high dose amphotericin and $\mathrm{G}-\mathrm{CSF}$, and receiving no further chemotherapy. He developed new skin lesions and his visual acuity declined.

A skin biopsy was performed, which showed signs of acute and chronic inflammation in association with fungal hyphae (fig 3). A repeat bone marrow examination was also performed, which confirmed that the patient's ALL was in continuing complete remission. Over the next few weeks the patient continued to deteriorate. Eventually, after receiving a dose of $3 \mathrm{mg} / \mathrm{kg}$ of Ambisome for 43 days, treatment was discontinued and palliative care initiated. The patient died five weeks later at home.

Postmortem examination showed appearances consistent with disseminated fungal infection, including multiple necrotic nodules in both lungs and both kidneys in addition to that seen previously. No residual leukaemia was seen.

\section{Discussion}

Fusarium species are saprophytic moulds that are ubiquitous in the soil and increasingly recognised as serious pathogens in the severely immunocompromised. Prolonged severe neutropenia $\left(<0.5 \times 10^{9}\right)$, corticosteroids, broad spectrum antibiotics, and indwelling intravenous catheters are all important risk factors for the development of disseminated fusariosis. ${ }^{1}$ Several reviews in the literature, including the large series by Boutati et al, highlight the very poor prognosis in the absence of recovery from myelosuppression. ${ }^{1-3}$ In this series of 43 patients with disseminated fusariosis, only 13 patients responded to treatment and all of these responders obtained neutrophil recovery and remission from their underlying disease. Of the 30 non-responders, only three were in disease remission at the end of infection and all were still neutropenic. ${ }^{1}$ Similar results were seen in a smaller series by Musa et $a .^{2}$ Seven patients developed fusarial infection during chemotherapy induced neutropenia. Two patients subsequently developed locally invasive infection, but following resolution of neutropenia made a recovery on antifungal treatment. The remaining five patients had persistent neutropenia and developed disseminated systemic fusariosis, which proved fatal.

The poor prognosis of disseminated disease highlights the importance of early recognition and treatment of localised disease. Reports suggest that the skin is one of the most common sites of presentation of fusarial infection: this was the case in more than $55 \%$ of patients in several literature reviews. ${ }^{1-6}$ Primary skin lesions included cellulitis at the site of onychomycosis or at a wound site. There was no evidence of fungal nail infection in our patient and no evidence of previous skin breakdown at the site of his primary lesion on the dorsum of his hand. Metastatic skin lesions reported included both subcutaneous nodules and ecthyema gangrenosum-like lesions, as seen in our patient. ${ }^{1}$

Distinguishing fusarium from other types of fungus, such as the more common aspergillus species, is also very important because of the poor sensitivity that fusarium often shows to amphotericin..$^{1-6}$ Therefore, any suspicious lesion should be biopsied urgently to aid rapid diagnosis. Fusarium has been grown from blood cultures in a high proportion of patients ( $45 \%)$ with disseminated disease, so blood cultures should also always be taken to facilitate the correct diagnosis. ${ }^{135}$ High dose liposomal amphotericin is the current recommended treatment and should be started without delay if fusarial infection is suspected in a patient with known risk factors. Because of the poor prognosis with continued neutropenia, G-CSF is usually added, as in our patient, to stimulate neutrophil recovery. ${ }^{12}$

Our patient died from overwhelming fungaemia despite complete remission from ALL, a normal neutrophil count, and an initial response to treatment. One explanation might be related to the poor central nervous system penetration of intravenous amphotericin preparations. Eradication of the fungal infection could not be achieved despite local intravitreal antifungal treatment. This may have allowed reseeding of the blood from the right vitreous with secondary dissemination. We have found no reports of a similar secondary relapse in the literature. In those few cases where patients have developed a second episode of fusarial infection, having apparently cleared an initial 
infection, it has been in the context of a further neutropenic episode. ${ }^{13}$

The early diagnosis of fusarium may be better facilitated in the future with the development of polymerase chain reaction (PCR) based techniques. The detection of fusarial DNA by PCR in spiked blood and tissue from a mouse model has recently been reported to have a high sensitivity. ${ }^{7}$ These techniques might also aid correct species identification, although as yet there is no information to suggest this alters prognosis. Fusarium solani is the most common species reported, $F$ dimerum being highly unusual, and contributing to less than $2 \%$ of cases. ${ }^{1-6}$ Current treatment remains disappointing, with amphotericin being the only available agent. Its activity is often limited against fusarial infection, although this can be improved by using high dose treatment with a lipid formulation. An improvement in treatment might be realised using one of the new investigational triazoles such as voriconazole or
SCH 56592, which have shown promising potential in the treatment of fusariosis according to in vitro and preliminary animal studies. ${ }^{12}$ This case demonstrates the urgent need for new agents.

1 Boutati E, Anaissie E. Fusarium - a significant emerging pathogen in patients with haematologic malignancy: ten pathogen in patients with haematologic malignancy: ten years' experience at a cancer centre and
management. Blood 1997;90:999-1008.

2 Musa MO, Eisa AA, Halim M, et al. The spectrum of fusarium infection in immunocompromised patients with haematological malignancies and in non-immunocompromised patients: a single institution experience over 10 years. Br f Haematol 2000;108:544-8.

3 Gamis AS, Gudnason T, Giebink GS, et al. Disseminated infection with fusarium in recipients of bone marrow transplants. Rev Infect Dis 1991;13:1077-88.

4 Hennequin C, Lavarde V, Poirot JL, et al. Invasive fusarium infections: a retrospective study of 31 cases. 7 Med Vet Mycol 1997;35:107-14.

5 Merz WG, Karp JE, Hoagland M, et al. Diagnosis and successful treatment of fusariosis in the immunocompromised cessful treatment of fusariosis in the
host. F Infect Dis 1998;158:1046-55.

6 Venditti M, Micozzi A, Gentile G, et al. Invasive Fusarium solani infections in patients with acute leukaemia. Rev Infect Dis 1988;10:653-60

7 Hue FX, Huerre M, Rouffault MA, et al. Specific detection of fusarium species in blood and tissues by PCR technique. f Clin Microbiol 1999;37:2434-38.

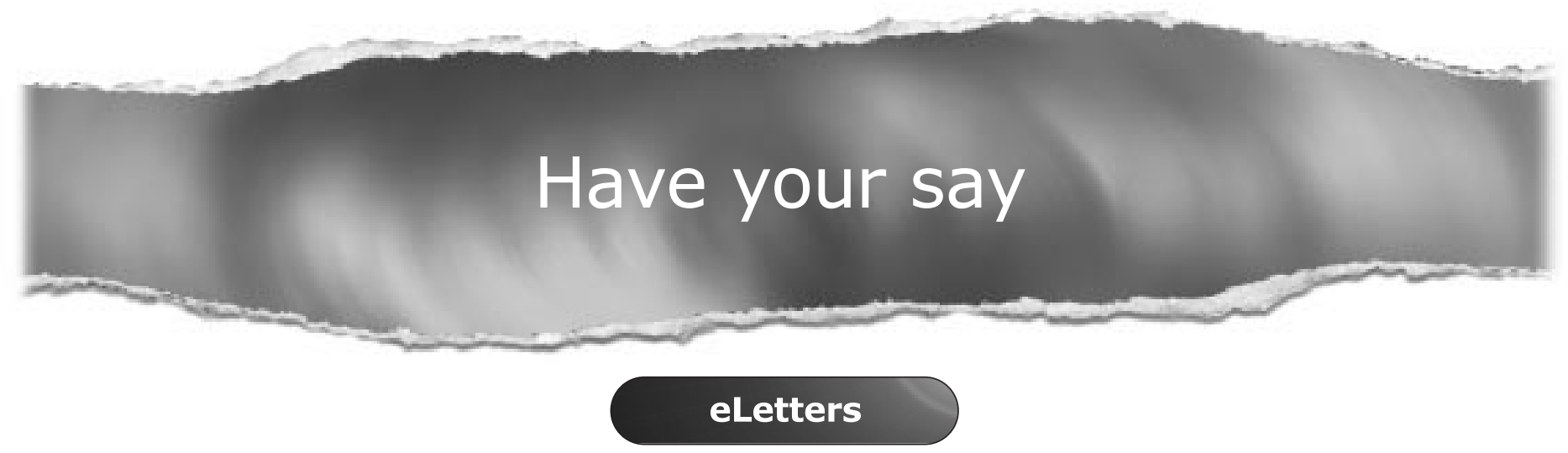

If you wish to comment on any article published in the Journal of Clinical Pathology you can send an eLetter using the eLetters link at the beginning of each article. Your response will be posted on Journal of Clinical Pathology online within a few days of receipt (subject to editorial screening).

\section{www.jclinpath.com}

